\title{
El consumo moderado de alcohol también disminuye el riesgo de accidentes cerebrovasculares
}

Light to moderate alcohol consumption and the risk of stroke among U.S. male physicians. Berger K, Ajani U, Kase C, et al. N Engl J Med 1999; 341:1557-64

\section{Objetivo}

Evaluar los efectos a largo plazo de distintos niveles de consumo de alcohol sobre el riesgo de padecer accidentes cerebrovasculares.

\section{Diseño}

Estudio de cohortes prospectivo con 12,2 años de seguimiento

Lugar

Estados Unidos

\section{Participantes}

22071 médicos de sexo masculino de entre 40 y 84 años, que participaban del Estudio de Salud de los Medicos (Physicians Health Study) ${ }^{1}$, sín antecedentes de accidentes cerebrovasculares, accidentes isquémicos transitorios y/o infarto de miocardio.

\section{Evaluación de los factores de riesgo}

Los participantes respondieron al comienzo del estudio un cuestionario, enviado por correo, con preguntas sobre factores de riesgo cardiovascular y frecuencia de consumo de alcohol. Este último punto según la cantidad de bebidas ingeridas, las posibles respuestas eran 1) menos de un vaso por semana 2) uno por semana 3) dos a cuatro por semana 4) cinco a seis por semana 5) uno o más por día. A los 84 meses de seguimiento el consumo de alcohol fue reevaluado usando el mismo cuestionario.

\section{Medición de los resultados principales}

Los eventos cardiovasculares (infarto de miocardio y accidentes cerebrovasculares), fueron evaluados por el cuestionario enviado por correo, el primer año cada seis meses y posteriormente en forma anual. Las muertes eran reportadas por los familiares o autoridades postales, y se verificaban por revisión de las historias clínicas disponibles y certificados de defunción. El diagnóstico de accidente cerebrovascular (ACV) se confirmaba, luego de una revisión de la información disponible en las historias clínicas por un comité conformado por expertos. Solo era considerado un primer episodio de ACV.

\section{Resultados principales}

Durante los 12,2 años de seguimiento fueron reportados 679 ACV que ocurrieron por primera vez: 557 isquémicos, 88 hemorrágicos, 34 sin categorizar. Para evaluar el efecto del consumo de alcohol sobre el riesgo de ACV se compararon los diferentes grupos de ingesta alcohólica con el grupo que reportó que raramente o nunca consumía alcohol. Se encontraron diferencias significativas en los grupos que consumían dos a cuatro ingestas por semana y más de una por día, RR 0,75 (IC95\% 0,58-0,96) y 0,8 (IC955 $0,64-0,99$ ) respectivamente. Al comparar el grupo que consumía menos de un vaso por semana con los que consumían por lo menos un vaso por semana se encontró luego de ajustar por potenciales confundidores una reducción del riesgo de $21 \%$ para ACV total y $23 \%$ para ACV isquémico, RR 0,79 (IC95\% 0,66-0,94) y RR 0,77 (IC95\% 0,63-0,94) respectivamente. No se encontró asociación entre consumo de alcohol y ACV hemorrágico.

\section{Conclusiones}

El consumo de alcohol bajo a moderado reduce significativamente el riesgo global de ACV y el riesgo de ACV isquémico. El beneficio se observa con un consumo tan bajo como el de un vaso por semana, un mayor consumo no confiere mayor protección.

Fuente de financiamiento: Institutos Nacionales de Salud (National Institutes of Health) y Servicio de Intercambio Académico Alemán.

\section{COMENTARIO}

Los accidentes cerebrovasculares son una importante causa de morbilidad y mortalidad en el mundo occidental, donde representan alrededor del $10 \%$ de las muertes totales 2 . La relación entre el consumo de alcoholy la disminución del riesgo de enfermedad coronaria es conocida, pero estudios de cohortes y caso control también sugieren un menor riesgo de ACV en individuos con un consumo moderado de alcohol. Si bien el presente estudio muestra una reducción del riesgo de ACV en los pacientes que consumen cantidades bajas y moderadas de alcohol existen sesgos y dificultades metodológicas que limitan la interpretación y generaLización de estos resultados.

Al analizar un estudio sobre alcohol y riesgo de eventos cardiovasculares debe hacerse un balance entre los efectos beneficiosos del alcohol con Los efectos deletéreos relacionados con su consumo. Las causas de muerte potencialmente relacionadas con el alcohol (muertes violentas; cirrosis; alcoholismo; cáncer de laringe, esófago, hígado y mama), no están reportadas en este estudio a pesar de que fueron evaluados y pueden encontrarse en otros artículos de la misma cohorte. Esta es una limitación ya que el beneficio real depende en gran medida de este equilibrio, en el mismo sentido probablemente ACV y enfermedad coronaria deberían ser evaluadas dentro del mismo artículo.

Las preguntas sobre el consumo de alcohol, están enfocadas hacia el consumo crónico y no son evaluados los episodios agudos de consumo (binge) que contribuyen a muertes violentas. Existe una marcada selección en la población estudiada que limita la validez externa; se trata de médicos hombres, lo que implica un nivel socio económico y cultural definido. La tasa de eventos cardiovasculares fue menor a la esperada por

\section{Referencias}

1. Final report on the aspirin component of the ongoing Physicians' Health Study. Steering Committee of the Physicians' Health Study Research Group. N Engl J Med. 1989 Jul 20:321(3):129-35.

2. Murray CJ, Lopez AD. GlobaL mortality, disability, and the contribution of risk factors: Global Burden of Disease Study. Lancet 1997; 349:1436-42

3. Potter J. Hazards and benefits of alcohol. N Engl J Med 1997;337:1763-1764

4. Hommel M. Alcohol for stroke prevention?. N Engl J Med 1999;341:1605-1606

5. Thun MJ, Peto R, Lopez AD, et al. Alcohol consumption and mortality among middle aged US adults. N.Engl. J. Med. $1997 ; 337: 1704-14$ los autores al comienzo del estudio; el consumo promedio de alcohol en la población general norteamericana es de 7,6 litros por persona por año, lo que equivale a dos vasos diarios, en el estudio la media de consumo fué menor a un vaso diario ${ }^{3}$. Un problema inherente a los estudios de cohortes es el hecho que al no existir una randomización de la intervención, la población expuesta puede estar protegida contra los eventos por otros factores conocidos o desconocidos. El aumento del HDL y un efecto positivo sobre el sistema fibrinolítico producido por el alcohol no explican completamente la reducción de los eventos cardiovasculares ${ }^{4}$. El efecto beneficioso puede estar vinculado a una conjunción de factores que configuren un hábito de vida del cual el consumo de alcohol sea solo la punta de un iceberg. Probablemente el alcohol dá protección contra los ACV, teniendo en cuenta que los resultados son similares a los reportados por Thun y colaboradores con una cohorte de 490000 personas de características basales similares a las de este estudio, en individuos que consumían menos de un vaso diario ${ }^{5}$. No hay duda que la única forma de poder dar respuesta a los aspectos oscuros mencionados es haciendo un estudio randomizado y controlado en prevención ecundaria dado que el beneficio es mayor cuando existe mayor riesgo cardiovascular, aunque esto puede estar limitado por cuestiones éticas. Mientras tanto debemos ser cautelosos, no hay ninguna sociedad científica reconocida que recomiende la prescripción de alcohol como un medicamento, dado el riesgo de muertes violentas, alcoholismo y cánceres relacionados, en todo caso debe ser evaluado por el médico en el consultorio según las características individuales de cada paciente y su riesgo cardiovascular.

Dr. Augusto Granel Unidad de fisiología clínica e hipertensión arterial. Hospital Italiano de Buenos Aires. 